1. RECHTSWISSENSCHAFT ALLGEMEIN / LAW IN GENERAL / SCIENCES JURIDIQUES EN GENERAL

S. $1-11$

1.1. Bibliographien / Bibliographies /

Bibliographies

S. $1-2$

1.2. Einführungen / Introductory works /

Introductions

1.3. Sammelwerke / Compilations / Ouvrages collectifs

1.4. Rechtsphilosophie / Legal philosophy / Philosophie du droit

S. $2-3$

S.

S. 3

1.5. Rechtssoziologie / Sociology of law / Sociologie juridique

S. $3-4$

1.6. Rechtsvergleichung / Comparative law / Droit comparé

S. 5

1.7. Rezeption / Reception / Réception

S. $5-9$

1.8. Ausbildung / Legal education / Enseignement

s. 9

1.9. Status der Frau / Women's status / Statut de la ferme

S. $9-11$

2. PRIVATRECHT / CIVIL LAW / DROIT CIVIL

S.11- 46

2.1. Privatrechtsgeschichte - Privatrecht allgemein / History of private law - Private law in general / Histoire du droit privé - Droit privé en général

$\mathrm{S} .11-14$

2.2. Allgemeiner Teil des Zivilgesetzbuches / General part of the Civil Code / Partie générale du code civil

$\mathrm{S} .14-15$

2.3. Schuldrecht / Obligations / Obligations

S. $15-19$

2.4. Sachenrecht / Property / Les biens

S. $19-21$

2.5. Familienrecht / Family law / Droit de la famille

S. $21-25$

2.6. Erbrecht / Law of inheritance / Successions

S. $25-26$

2.7. Handelsrecht / Commercial law / Droit commercial

s. $26-30$

2.8. Gesellschaftsrecht / Business organization/ Sociétés

$5.30-33$

2.9. Genossenschaften / Cooperatives / Coopératives

s. $33-34$

2.10. Wertpapierrecht / Negotiable instruments / Titres négociables

S. $\quad 34$

2.11. Gewerblicher Rechtsschutz - Urheber- und Verlagsrecht / Industrial property - Copyright and publishing law / Propriété industrielle - Droit d'auteur et d'édition 
2.12. Wettbewerbs- und Warenzeichenrecht / Unfair competition - Trademarks / Concurrence déloyale - Marques de fabrique

2.13. Internationales Privatrecht / Conflict of laws / Droit international privé

3. GERICHTSVERFASSUNG - ALLGEMEINES PROZESSRECHT ZIVILPROZESS / JUDICIAL SYSTEM - PRACTICE AND PROCEDURE - CIVIL PROCEDURE / ORGANISATION JUDICIAIRE - DROIT JUDICIAIRE EN GENERAL PROCEDURE CIVILE

3.1. Allgemeines / In general / En général

3.2. Gerichtsverfassung / Judicial system / organisation judiciaire

3.3. Richter und Staatsanwälte / Judges and public prosecutors / Juges et procureurs

3.4. Allgemeines Prozeßrecht - ZivilprozeB / Practice and procedure - Civil procedure / Droit judiciaire en général - Procedure civile

3.5. Schiedsgerichtsbarkeit / Arbitration / Arbitrage

3.6. Zwangsvollstreckung - Konkursrecht / Enforcement of judgements - Bankruptcy / Exécution forcée - Faillite

S. $52-53$

3.7. Notar / Notary / Notaire

S. 53

3.8. Personenstandsrecht / Civil status registration / Registre d'état civil

S. $\quad 53$

4. STRAFRECHT / CRIMINAL LAW / DROIT PENAL S.54 - 59

4.1. Allgemeines / In general / En général S.54 - 56

4.2. Strafgesetzbuch, Allgemeiner Teil / Criminal Code, general part / Partie générale du Code pénal

S. 56

4.3. Strafgesetzbuch, Besonderer Teil / Criminal Code, particular part / Partie particulaire du Code pénal

S. $56-57$

4.4. StrafprozeBrecht / Criminal procedure / Procédure pénale

S.

4.5. Jugendstrafrecht / Juvenile offenders / Droit pénal spécial des mineurs délinquants

s.

4.6. Wehrstrafrecht / Military offences / Droit pénal militaire

S. $\quad 58$

4.7. Strafvollzug / Penology / Science pénitentiaire

S. $\quad 59$

4.8. Internationales Strafrecht / International criminal law / Droit international pénal

S. 
5. STAATSLEHRE - POLITIK / GENERAL THEORY OF STATE POLITICS / THEORIE DE L'ETAT - POLITIQUE

S. $59-78$

5.1. Allgemeines / In general / En général

S. $59-68$

5.2. Politische Ideologien und ordnungssysteme / Political ideologies and political systems / Idéologies politiques et systèmes socials

5.3. Parteien / Political parties / Partis politiques

5.4. Wahlen / Elections / Elections

S. $73-76$

6. STAATS- UND VERFASSUNGSRECHT / CONSTITUTIONAL LAW / DROIT CONSTITUTIONNEL

S. $76-78$

$5.78-89$

6.1. Allgemeines zum రffentlichen Recht / Public law in general / Droit public en général

S. 78

6.2. Staats- und Verfassungsrecht allgemein / Constitutional law in general / Droit constitutionnel en général

6.3. Verfassungsentwicklung bis 1924 / Constitutional development until 1924 / Développement constitutionnel jusqu'à 1924

S. $80-82$

6.4. Verfassung vom 20.April 1924 / Constitution of 20 april 1924 / Constitution de 20 avril 1924

S. $82-83$

6.5. Die Revolution vom 27. Mai 1960 / The revolution of 27 th of may $1960 /$ La révolution de 27 mai 1960

$\mathrm{S} .83-84$

6.6. Die Verfassung vom 9.Juli 1961 / The constitution of 9 th of july 1961 / La constitution de 9 juillet 1961

S. $84-85$

6.7. Die Verfassungsreform von 1971 / The constitutional reform of 1971 / La réforme constitutionnelle de 1971

S. $85-86$

6.8. Der Putsch vom 12.September 1980 und die Verfassungsentwicklung / The coup d'etat of 12 th of september 1980 and the constitutional development / Le coup d'état de

12 septembre et le développement constitutionnel

s. 86

6.9. Grundfragen der verfassungsstaatlichen ordnung / Fundamental Aspects of Constitutionalism / Aspects fondamentals du constitutionalisme

6.10. Grund- und Menschenrechte / Civil and human rights / Libertés publiques - Droits de 1 'homme

6.11. Verfassungsgerichtsbarkeit / Constitutional courts / Juridiction constitutionnelle 
7. ALLGEMEINES UND BESONDERES VERWALTUNGSRECHT / ADMINSITRATIVE LAW - ADMINISTRATIVE LAW (INDIVIDUAL BRANCHES) / DROIT ADMINISTRATIF - DROIT ADIIINISTRATIF (BRANCHES INDIVIDUELLES)

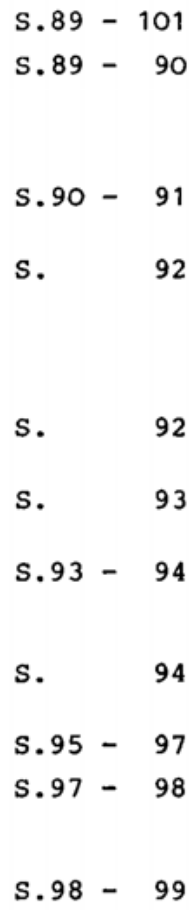

s.

s.

\section{S.93- 94}

s.

7.1. Allgemeines / In general / En général

7.2. Verwaltungsorganisation - Verwaltungsreform / Administrative organization - Administrative reform / Organisation administrative Reforme administrative dure / Procédure administrative

7.4. Amtshaftung - Enteignung - రffentlich-rechtliche Entschädigung / Liability of the stateEminent domain or expropriation - Law of public compensation / Responsabilité de l'état Expropriation - Dommages-intérêts

7.5. Verwaltungsgerichtsbarkeit / Administrative courts / Juridiction administrative

7.6. రffentlicher Dienst / Civil service / Service publique

7.7. Polizei- und ordnungsrecht / Police regulations - Public security and order / Securité et ordre public - Police

7.8. Kommunalrecht / Local government / Droit municipal

7.9. Bildungswesen / Education / Enseignement

7.10. Presse-, Rundfunk-, Film- und Fernsehrecht / Press - Broadcasting - Film - Television/ Presse - Radio - Cinéma - Télévision

7.11. Raumordnung - Städteplanung / Regional planning - Town planning / Aménagement du territoire - Aménagement urbain

S.99- 100

7.12. ర̈ffentliches Grundstücks-, Bau- und Wohnungsrecht / Zoning, building and housing regulations / Droit foncier public - Construction immobilière - Lotissements

\section{S.}

S.100- 101

Straßen-, und Wegerecht / Highways and roads / Voies pubiliques

S. 101

7.14 Unwelt-, Natur- und Landschaftsschutz /

Environmental protection - Nature protectionPreservation of landscape / Protection de la nature et de la paysage

s. 101

8. WIRTSCHAFTSRECHT / ECONOMIC LEGISLATION / DROIT ECONOMIQUE

$\mathrm{S} .101-111$

8.1. Allgemeines / In general / En général

S. $101-102$

8.2. Wirtschaftsverfassung / Legal framework of economic system / Structure économique

S. 102

8.3. Wirtschaftsverwaltung und - organisation / 
Public organization of economy / Adroinistration et organisation économique $\mathrm{S}$

S. $\quad 102$

8.4. Außenwirtschaftsrecht / Foreign trade regulations / Commerce international - Economie extérieur

S. $102-104$

8.5. Verbraucherschutz / Consumer protection / Protection du consommateur

S. 104

8.6. Geld - Kredit - währung - Banken - Börsen / Money - Credit - Currency - Banking - Stock exchanges / Argent - Crédit - Banques Bourses

8.7. Gewerbe - Handwerk - Industrie / Trades Crafts - Industries / Commerce - Corps de métier - Industrie

s. 106

8.8. Bergrecht - Energierecht / Mining - Energy / Droit minier - Energie

S. 107

8.9. Wasserrecht - Public waters - Droit d'eaux

s. 107

8.10. Landwirtschaft - Forst- und Jagdrecht / Agriculture - Forest and game laws / Droit agraire - Droit forestier - Droit de chasse

S.107- 109

8.11. Investitionsförderung / Investment encouragement / Encouragement de l'investissement

S. $109-110$

8.12. Joint Ventures / Joint ventures / Joint ventures

S. 111

9. VERKEHRSRECHT / TRAFFIC LAWS / DROIT DE CIRCULATION

$\begin{array}{lr}\text { s.111 } & -112 \\ \text { s. } & 111 \\ \text { s. } & 111 \\ \text { s. } & 111 \\ \text { s. } & 112 \\ \text { s. } & 112 \\ \text { s. } & 112\end{array}$

9.1. Allgemeines / In general / En général

9.2. Straßenverkehr / Road traffic / Circulation routière

10. FINANZ- UND STEUERRECHT / FINANCIAL LAWS AND TAXATION / DROIT FINANCIER ET FISCAL

S.113- 116

10.1. Allgemeines / In general / En général

s. 113

10.2. Finanzverfassung / Financial organization / Organisation financière

S,

10.3. Finanzverwaltung / Tax administration / Administration fiscale

10.4. Haushaltsrecht / Budget / Budget S

S. 113

10.5. Staatsschulden / Public debts / Dette publiques. 114

10.6. Steuerrecht / Tax law / Droit fiscal S.114 - 116

10.7. Zollrecht / Customs law / Droit douanière $S$. 116 
10.8. Internationales Steuerrecht / International taxation / Droit international fiscal

S. 116

11. ARBEITSRECHT / LABOR LAW / DROIT DU TRAVAIL

11.1. Allgemeines / In general / En général

11.2. Arbeitsvertragsrecht / Contracts of employment / Contrat de travail

11.3. Kollektives Arbeitsrecht - Tarifvertragsrecht / Organized Labor - Collective bargaining / Relations collectives du travail Droit des conventions collectives

$\mathrm{S} .119-122$

11.4. Betreibsverfassung - Mitbestimmung / Works council - Co-participation of labour / Relations direction-personnel - Cogestion

S.

11.5. Arbeitsschutz / Protection of employees / Protection du travail

$\mathrm{S} .122-123$

11.6. Arbeitsmarkt - Arbeitsverwaltung - Arbeitslosenversicherung / Employment market public labor administration - Unemployment insurance / Marché du travail - Bureaux de placement - Assurance-chômage

S.

12. SOZIALRECHT / SOCIAL LAW / DROIT SOCIAL

$\mathrm{S} .123-129$

12.1. Allgemeines / In general / En général

S. $123-125$

12.2. Sozialpolitik / Social policy / Politique sociale

s.

12.3. Sozialhilfe - Soziale Sicherheit / Public welfare - Social security / Assistance publique - Securité sociale

S.

12.4. Allgemeines zum Sozialversicherungsrecht/ Social insurance in general / Securité sociale en générale

S. $125-127$

$\mathrm{S} .127-128$ Assurance-maladie

12.6. Unfallversicherung / Accident insurance / Assurance contre les accidents

s.

12.7. Rentenversicherung / Annuity and disability insurance / Assurance-rentes

S.

12.8. Privatversicherungsrecht / Private insurance/ Assurances privées

S.

12.9. Internationales Sozialrecht / International social legislation

s.

13. VÖLKERRECHT UND INTERNATIONALE POLITIK / PUBLIC INTERNATIONAL LAW AND INTERNATIONAL RELATIONS / DROIT INTERNATIONAL PUBLIC ET RELATIONS INTERNATIONALES

13.1. Allgemeines / In general / En général

S. $129-199$

S. $129-130$

13.2. Staaten und Räume im Völkerrecht / States and territories / Etats et territoires

S. 130 
13.3. Internationale Beziehungen / International : relations / Relations internationales

13.4. Internationale Verträge / International treaties / Traités internationals

13.5. Kapitulationen / Capitulations / Capitulations

13.6. Aufteilung des Osmanischen Reiches / Partition of the Ottoman Empire / Démembrement de 1 'Empire Ottoman

13.7. Vertrag von Sèvres - Meerengenfrage / Treaty of Sèvres - The Straits question / Traité de Sèvres - La question des Détroits

s. 150

13.8. Vertrag von Lausanne - Meerengenfrage - Bevölkerungsaustausch / Treaty of Lausanne The Straits question - Exchange of populations / Traité de Lausanne - La question des Détroits - Echange des populations

13.9. Mossulfrage / The Mosul question / La question de Mossoul

13.10.Vertrag von Montreux - Meerengenfrage / Treaty of Montreux - The Straits question/ Traité de Montreux - La question des Détroits S.154 - 159

13.11.Der Streit um den Festlandsockel in der Ägäis / Aegean Sea Continental Shelf Case / Affaire du plateau continental de la mer Egée $\quad$ S.159 - 164

13.12.zypernkonflikt / The Cyprus Problem / Le conflit de Chypre

13.13.Staatsangehörigkeitsrecht / Nationality / Nationalité

13.14. Ausländerrecht / Aliens / Etrangers / Flüchtlinge / Refugees / Refugiés

13.15.Minderheiten / Minorities / Minorités

13.16. Internationale organisationen / International Organizations / Organisations internationales S.

13.17. Beziehungen zwischen der Türkei und der EG / Relations between Turkey and the EC / Relations entre la Turquie et la CE $\quad$ S.197 - 199

13.18. Internationale Gerichtsbarkeit / International Adjudication / Juridiction inetrnationale s.

Verfasser- und Herausgeberverzeichnis 
